\title{
web cellHTS2: A web-application for the analysis of high-throughput screening data
}

\author{
Oliver Pelz, Moritz Gilsdorf and Michael Boutros*
}

\begin{abstract}
Background: The analysis of high-throughput screening data sets is an expanding field in bioinformatics. Highthroughput screens by RNAi generate large primary data sets which need to be analyzed and annotated to identify relevant phenotypic hits. Large-scale RNAi screens are frequently used to identify novel factors that influence a broad range of cellular processes, including signaling pathway activity, cell proliferation, and host cell infection. Here, we present a web-based application utility for the end-to-end analysis of large cell-based screening experiments by cellHTS2.

Results: The software guides the user through the configuration steps that are required for the analysis of single or multi-channel experiments. The web-application provides options for various standardization and normalization methods, annotation of data sets and a comprehensive HTML report of the screening data analysis, including a ranked hit list. Sessions can be saved and restored for later re-analysis. The web frontend for the cellHTS2 R/Bioconductor package interacts with it through an R-server implementation that enables highly parallel analysis of screening data sets. web cellHTS2 further provides a file import and configuration module for common file formats.

Conclusions: The implemented web-application facilitates the analysis of high-throughput data sets and provides a user-friendly interface. web cellHTS2 is accessible online at http://web-cellHTS2.dkfz.de. A standalone version as a virtual appliance and source code for platforms supporting Java 1.5.0 can be downloaded from the web cellHTS2 page. web cellHTS2 is freely distributed under GPL.
\end{abstract}

\section{Background}

High-throughput cell-based screens have become an important experimental tool for the analysis of many cellular processes. Whole genome sequences and methods for gene silencing by RNA interference (RNAi) have enabled loss-of-function analysis in ex vivo and in vivo, opening new avenues for functional analysis that were previously unfeasible $[1,2]$. Different experimental methods to assess phenotypic changes are being used, from single-channel homogenous readouts to multi-channel cytometry and imaging, producing large data sets that need to be analyzed to extract phenotypically relevant information. RNAi screening has found a broad user-base as a genetic method to dissect many different cellular

*Correspondence: m.boutros@dkfz.de

${ }^{1}$ German Cancer Research Center (DKFZ), Div Signaling and Functional

Genomics and University of Heidelberg, Faculty of Medicine Mannheim, Dept Cell and Molecular Biology, Im Neuenheimer Feld 580, D-69120 Heidelberg, Germany

Full list of author information is available at the end of the article processes, such as cell survival, signaling pathways and other cellular phenotypes in a high-throughput manner [3-6].

High-throughput screens are mostly performed using 96- to 384-well plates and produce large data sets that need to be normalized, summarized and ranked to generate a list of significant phenotypic modifiers. Large-scale RNAi screens can easily exceed more than 100,000 data points per screening experiment and specialized statistical approaches have been developed for their analysis [710]. Quality control assessments of assays and screening data are performed to provide benchmarks for the overall performance, such as experiment-wide performance of controls, reproducibility between replicate experiments, as well as other statistical quality control measures [1013].

We have previously described cellHTS as an analysis toolbox for cell-based high-throughput screens [7]. cellHTS is implemented in R/Bioconductor [12] as a 
command-line utility that provides a workflow for the analysis of high-throughput data sets. cellHTS and cellHTS2 have become widely used in the community as they provide an end-to-end solution for the analysis of high-throughput screening data sets, while retaining the flexibility to incorporate further functions for statistical analysis as the field matures. However, an obstacle for general use in the laboratory was the lack of an integrated and easy-to-use solution for the configuration of screening plates, choice of controls and analysis methods.

Here, we present a web-based application that guides the user through all steps required for the analysis of high-throughput screens, including configuration of

\section{Selection of assay type}
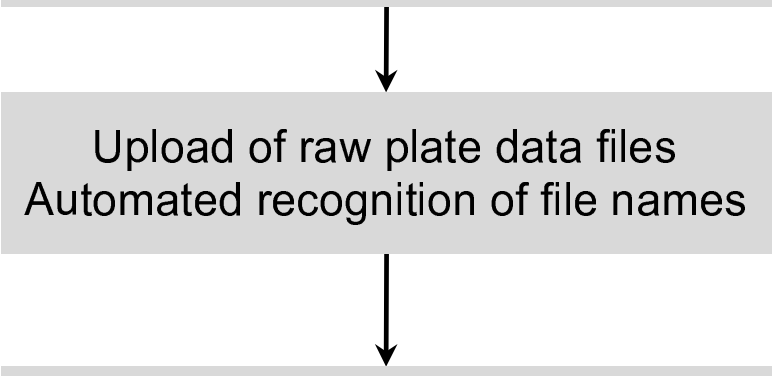

Plate configuration with positive, negative controls, sample wells

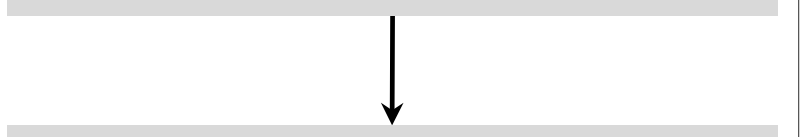

\section{Selection of standardization and} normalization methods

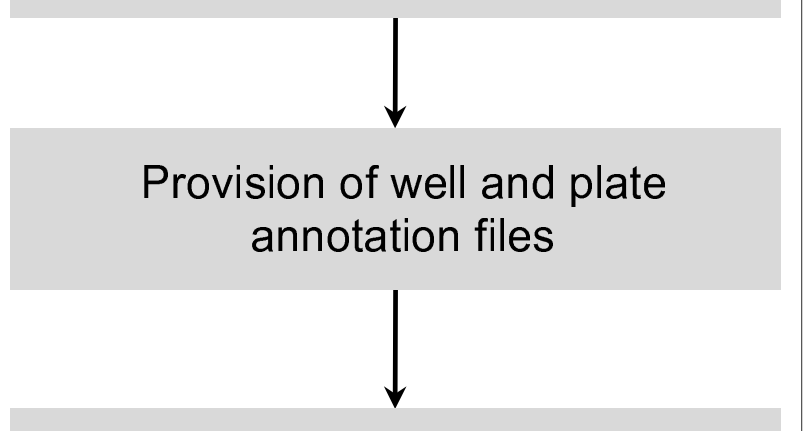

Analysis report as HTML

Figure 1 Schematic representation of the workflow for the analysis of high-throughput screening data sets. plates and controls, normalization and statistical quality control (Figure 1). The software allows the user to upload a variety of file formats, select different methods for summarization and normalization, and returns a complete analysis report by E-mail or as a download. Analysis workflows can be saved as templates for re-analysis, or possible submission as supplementary material for publications. The software can be accessed at http://webcellHTS2.dkfz.de or downloaded as a fully functional Java application (for platforms supporting Java 1.5.0) or VirtualBox appliance.

\section{Implementation}

Data files needed for the analysis are generated through the graphical user interface or can be provided through upload forms. web cellHTS2 also provides an import module that supports upload of a spectrum of different file formats. web cellHTS2 implements error detection mechanisms for each data file or website input, checking for common input errors prior to running cellHTS2. Once the configuration of a screening experiment is completed, the analysis project, containing information on the complete session including all input files and processing parameters, can be saved for re-use. This function allows for rapid re-processing of similar datasets and generation of a full documentation of the analysis. The results of the analysis can be streamed to the web browser or can be sent via E-mail directly.

web cellHTS2 was implemented based on a Java Server Pages infrastructure using the Tapestry5 [14] opensource web-framework, which facilitates maintenance and extension of the web application. The frontend has been designed to run remotely on a Tomcat 5 webserver [15] but can also be installed locally using an integrated Jetty [16] Java web-server. AJAX (Asynchronous JavaScript and XML) technology is used to improve the interactivity of web cellHTS2.

Interaction between the cellHTS2 R/Bioconductor software and the Java based web application is achieved using R-serve, which can be run on an independent webserver. This separates the web application logic from the statistical calculations, thereby reducing the computational load for accessing the webserver and allowing the analysis of several high-throughput screening data sets in parallel (Figure 2). The workload for computational calculations can be scaled at runtime by setting the maximum number of parallel analyses.

\section{Results}

web cellHTS2 facilitates the analysis of high-throughput screening data by providing an easy to use web-application. It has been developed with a view towards largescale RNAi screens but can also be employed for the analysis of small molecule screens. A particular focus has 


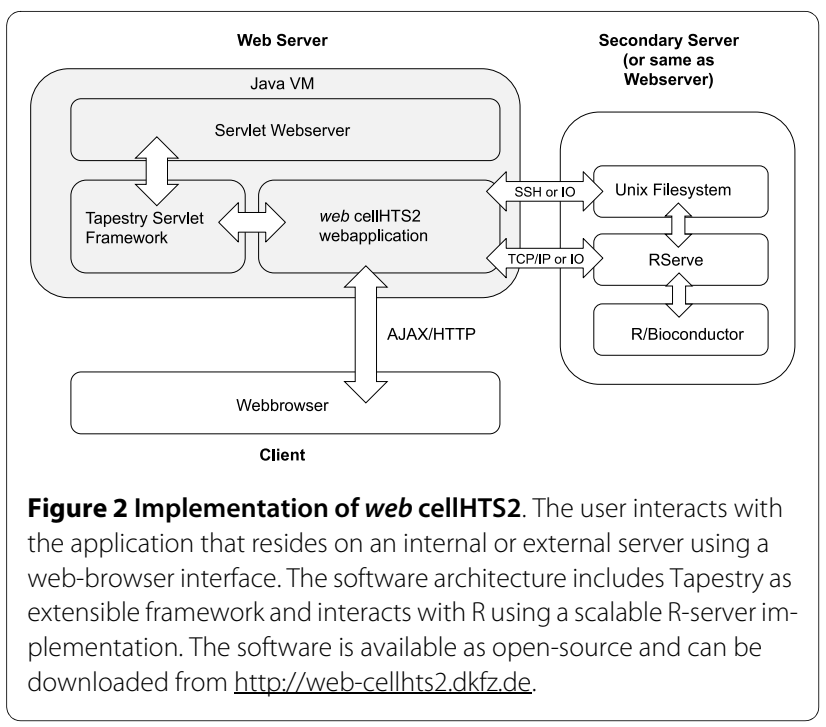

been to provide a user-friendly interface to select analysis parameters and to generate "re-usable" analysis workflows. Furthermore, error-checking procedures of raw data and annotation files, and automated pre-processing of uploaded data have been implemented. web cellHTS2 can be accessed online or downloaded for local installation. web cellHTS can also be downloaded as a virtual appliance to run web cellHTS2 in a contained environment [17].

The web application implements three steps in the analysis workflow. In the first step, the user starts a new analysis by choosing the type of experiment (current options are single or dual-channel experiments) or can upload a previously saved workflow (Figure 3a). Uploaded workflows can be modified, e.g. by altering analysis parameters or replacing data files. In the next step, raw data files from high-throughput screening experiments are uploaded. The web application recognizes plate, replicate and channel files, or file assignments can be manually annotated. This feature allows rapid upload of large data sets that can easily entail several hundred data files (such as generated by multi-mode platereaders). Also, previously generated "plate list" files can be uploaded (Figure 3b). An import module can also be used to upload more complex data files. Step three involves configuration of the layout of the multi-well plates which were used in the screening experiment. The graphical user interface allows the user to indicate which wells contain negative, positive and other controls (Figure 3c). Alternatively, previously generated configuration files with existing plate layouts can be uploaded. At this stage the user can select among multiple options regarding how the data is processed and different channels summarized. Table 1 shows a list of options that are currently implemented in cellHTS2, which include both sample and control-based normalization methods, as well as procedures such as B-score normalization [18] to remove spatial artefacts. In the last step, annotation and description files are uploaded or manually described using an edit form. The workflow can then be saved for future analysis or reference. The analysis report is either streamed as a compressed file via HTML or sent by Email. A tutorial on the analysis of high-throughput screens with web cellHTS2, including a sample data set, is provided on the website.

\section{Discussion}

The application presented here is a web or stand-alone program to facilitate the analysis of high-throughput screening data. High-throughput screening experiments are of increasing importance, both for basic science and drug discovery. Such data sets easily exceed the complexity of transcriptome experiments, however there are still comparably much fewer tools available that enable an easy-to-use analysis. cellHTS and other software packages [9] have started to address this issue by enabling an end-to-end analysis of high-throughput screening data sets and have become widely used in the community. Here, we provide a web application as a front-end for cellHTS2 to increase its accessibility and accelerate the analysis of high-throughput screening data sets. The web application can be used both for RNAi and compound screening experiments and can be extended to meet future needs. In contrast to commercial packages, we provide an open-source and extensible solution for online and offline usage.

\section{Conclusions and future directions}

web cellHTS2 provides an intuitive interface for the analysis of high-throughput screens. The user can choose among different options for the analysis of screening data sets. Statistical analysis options will be expanded as new methods become available and broadly used $[9,18]$. The graphical user interface for the configuration of screening experiments and the option to save "re-usable" session templates make it convenient to use in the laboratory. Future developments of the application will be to provide direct links to phenotype databases [19], e.g. to compare hit lists, to annotate hit list with additional information from public databases e.g. through BioMart and to extend the analysis by functional annotation data such GO enrichment analysis. It is also planned to provide diagnostic plots "on-the-fly" to allow the user to compare different normalization strategies.

\section{Availability and requirements}

Project name: web cellHTS2

Project home page: http://web-cellHTS2.dkfz.de 


\section{a web cellHTS2 \\ Introduction \\ web cellHTS2 is a front end for cellHTS2 for an end-to-end analysis of cell-based high-throughput screening experiments. The onlin analysis options and outputs a HTML including a full quality control report and a ranked hitlist. A downloadable package for local installation can be found here. \\ cellHTS2 is a software package implemented in Bioconductor/R for cell-based high-throughput RNAi screens. The cellHTS2 package is A set of example files can be downloaded from the online tutorial here. Additional sample files can also be found in the latest cellHTS source package (in source folder: inst) here. \\ Start of a web celliHTS2 session \\ Load previous session or start a new session (download example session ling \\ (For more information click here) \\ Step 1: Select type of experiment \\ Dual Channel \\ Would you like to label your channels?

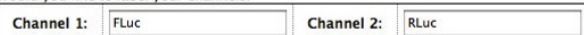 \\ \begin{tabular}{|l|l|l|}
\hline [ back ] & [ restart ] & [nex] \\
\hline
\end{tabular}}

b

Step 2: Upload and assign data files to channels and replicates (help).

Data Files can be uploaded as raw plate reader, text files in cellHTS2 format or ZIP archives Filelmporter module can be used for more complex file types (help).

Advanced File Importer

\section{Browse...}

Filename Parsing: $\square$

File Name $\odot$ Plate Number $\odot$ Replicate $\odot$ Channel $\odot$

\begin{tabular}{|l|l|l|l|}
\hline RA01D1.TXT & 1 & 1 & 1 \\
\hline RA01D2.TXT & 1 & 2 & 1 \\
\hline RA02D1.TXT & 2 & 1 & 1 \\
\hline RA02D2.TXT & 2 & 2 & 1 \\
\hline RA03D1.TXT & 3 & 1 & 1 \\
\hline RA03D2.TXT & 3 & 2 & 1 \\
\hline RB01D1.TXT & 1 & 1 & 2 \\
\hline RB01D2.TXT & 1 & 2 & 2 \\
\hline RB02D1.TXT & 2 & 1 & 2 \\
\hline RB02D2.TXT & 2 & 2 & 2 \\
\hline RB03D1.TXT & 3 & 1 & 2 \\
\hline RB03D2.TXT & 3 & 2 & 2 \\
\hline
\end{tabular}

C

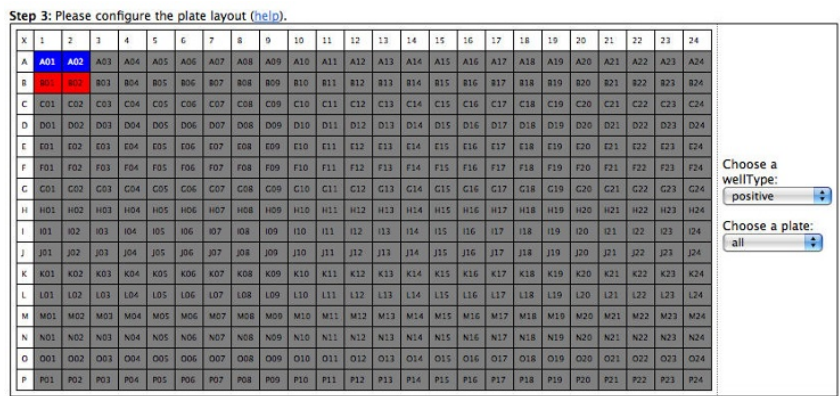

and/or Upload Plate Config file (help).
Browse..

and/or Upload Screenlog file (help).

Figure 3 Screenshots of the analysis workflow of high-throughput screens by web cellHTS2. (a) The user can start a new analysis or upload previous analysis templates. (b) The data file upload form with parameter editor. (c) Graphical plate configuration editor. 
Table 1: Examples of normalization options

\begin{tabular}{|c|c|}
\hline Normalization option & Description \\
\hline Median & $\begin{array}{l}\text { Measurements are divided } \\
\text { by the median of all sample } \\
\text { wells in the plate }\end{array}$ \\
\hline Shorth & $\begin{array}{l}\text { The midpoint of the 'shorth' } \\
\text { of the distribution of all } \\
\text { sample wells is used for } \\
\text { normalization }\end{array}$ \\
\hline Mean & $\begin{array}{l}\text { Measurements are divided } \\
\text { by the mean of all sample } \\
\text { wells in the plate }\end{array}$ \\
\hline Negatives & $\begin{array}{l}\text { Measurements are divided } \\
\text { by the median of negative } \\
\text { controls in the plate }\end{array}$ \\
\hline Percent control & $\begin{array}{l}\text { Measurements are divided } \\
\text { by the mean of the plate's } \\
\text { positive control }\end{array}$ \\
\hline Normalized percent control & $\begin{array}{l}\text { Measurements are divides by } \\
\text { the difference of the plates } \\
\text { positive and negative } \\
\text { controls }\end{array}$ \\
\hline B-score & $\begin{array}{l}\text { A two-way (row and column) } \\
\text { median polish is applied to } \\
\text { each plate }\end{array}$ \\
\hline Robust local fit regression & $\begin{array}{l}\text { Spatial effects are normalized } \\
\text { by fitting a bivariate local } \\
\text { regression }\end{array}$ \\
\hline Loess regression & $\begin{array}{l}\text { Spatial effects are normalized } \\
\text { using Loess regression }\end{array}$ \\
\hline
\end{tabular}

Operating system(s): Platform independent Programming language: e.g. Java Other requirements: Java 1.5.0

Downloadable Version: R 2.10.0, cellHTS2 2.11.1 and

\section{Rserve 0.6.0}

Virtual appliance: Open source software Virtual box http://www.virtualbox.org

License: GNU GPL Any restrictions to use by non-academics: none

\section{Competing interests}

The authors declare that they have no competing interests.

\section{Authors' contributions}

OP developed the software. MG provided advice in the design and development of the software package. MB conceived the concept and methodology and supervised the project. MB and OP wrote the manuscript. All authors read and approved the final manuscript.

\section{Acknowledgements}

We thank Grainne Kerr, Xian Zhang, Gregoire Pau and Wolfgang Huber for helpful suggestions and critical comments on the manuscript. We are grateful to Tobias Reber for help with IT infrastructure. Funding was provided by grants from the Helmholtz Alliance for Systems Biology and BMBF NGFN-Plus (01GS08181)

\section{Author Details}

German Cancer Research Center (DKFZ), Div Signaling and Functional Genomics and University of Heidelberg, Faculty of Medicine Mannheim, Dept Cell and Molecular Biology, Im Neuenheimer Feld 580, D-69120 Heidelberg, Germany

Received: 3 October 2009 Accepted: 12 April 2010

Published: 12 April 2010

\section{References}

1. Boutros $M$, Ahringer J: The art and design of genetic screens: RNA interference. Nature Reviews Genetics 2008, 9:554-566.

2. Moffat J, Sabatini DM: Building mammalian signalling pathways with RNAi screens. Nature Reviews Molecular Cell Biology 2006, 7:177-187.

3. Flockhart I, Booker M, Kiger A, Boutros M, Armknecht S, Ramadan N, Richardson K, Xu A, Perrimon N, Mathey-Prevot B: FlyRNAi: the Drosophila RNAi screening center database. Nucleic Acids Research 2006, 34:D489-494.

4. Bartscherer K, Pelte N, Ingelfinger D, Boutros M: Secretion of Wnt ligands requires Evi, aconserved transmembrane protein. Cell 2006, 125:523-533.

5. Bard F, Casano L, Mallabiabarrena A, Wallace E, Saito K, Kitayama H, Guizzunti G, Hu Y, Wendler F, Dasgupta R, Perrimon N, Malhotra V: Functional genomics reveals genes involvedin protein secretion and Golgi organization. Nature 2006, 439:604-607.

6. Huang S, Laoukili J, Epping MT, Koster J, Hölzel M, Westerman BA, Nijkamp W, Hata A, Asgharzadeh S, Seeger RC, Versteeg R, Beijersbergen RL, Bernards R: ZNF423 is criticallyrequired for retinoic acid-induced differentiation and is a marker of neuroblastoma outcome. Cancer Cell 2009, 15:328-340.

7. Boutros M, Bras LP, Huber W: Analysis of cell-based RNAi screens. Genome Biology 2006, 7:R66.

8. Birmingham A, Selfors $L M$, Forster T, Wrobel D, Kennedy CJ, Shanks E, Santoyo-Lopez J, Dunican DJ, Long A, Kelleher D, Smith Q, Beijersbergen RL, Ghazal P, Shamu CE: Statistical methods for analysis of highthroughput RNA interference screens. Nature Methods 2009, 6:569-75.

9. Rieber N, Knapp B, Eils R, Kaderali L: RNAither, an automated pipeline for the statisticalanalysis of high-throughput RNAi screens. Bioinformatics 2009, 25:678-9.

10. Chung N, Zhang XD, Kreamer A, Locco L, Kuan PF, Bartz S, Linsley PS, Ferrer M, Strulovici B: Median absolute deviation to improve hit selection for genome-scale RNAi screens. Journal of Biomolecular Screening 2008, 13:149-58.

11. Zhang JH, Chung TD, Oldenburg KR: A Simple Statistical Parameter for Use in Evaluation and Validation of High Throughput Screening Assays. Journal of Biomolecular Screening 1999, 4:67-73.

12. Gentleman RC, Carey VJ, Bates DM, Bolstad B, Dettling M, Dudoit S, Ellis B, Gautier L, Ge Y, Gentry J, Hornik K, Hothorn T, Huber W, lacus S, Irizarry R, Leisch F, Li C, Maechler M, Rossini AJ, Sawitzki G, Smith C, Smyth G, Tierney $L$, Yang JY, Zhang J: Bioconductor: opensoftware development for computational biology and bioinformatics. Genome Biology 2004, 5:R80.

13. Malo N, Hanley JA, Cerquozzi S, Pelletier J, Nadon R: Statistical practice in high-throughput screening data analysis. Nature Biotechnology 2006, 24:167-75.

14. Apache Tapestry 5 [http://tapestry.apache.org] 
15. Apache Tomcat 5.5 [http://tomcat.apache.org]

16. Jetty Java webserver [http://www.mortbay.org/jetty/]

17. Virtual Box [http://www.virtualbox.org/]

18. Brideau C, Gunter B, Pikounis B, Liaw A: Improved statistical methods for hit selection inhigh-throughput screening. Journal of Biomolecular Screening 2003, 8:634-647.

19. Horn T, Arziman Z, Berger J, Boutros M: GenomeRNAi: a database for cellbased RNAiphenotypes. Nucleic Acids Research 2007, 35:D492-7.

doi: 10.1186/1471-2105-11-185

Cite this article as: Pelz et al., web cellHTS2: A web-application for the analysis of high-throughput screening data BMC Bioinformatics 2010, 11:185

Submit your next manuscript to BioMed Central and take full advantage of:

- Convenient online submission

- Thorough peer review

- No space constraints or color figure charges

- Immediate publication on acceptance

- Inclusion in PubMed, CAS, Scopus and Google Scholar

- Research which is freely available for redistribution

Submit your manuscript at www.biomedcentral.com/submit 